\title{
Characterization of Nickel Oxide Nanoplates Using STEM Tomography and In- situ Electron Microscopy
}

\author{
A.K. Shukla, ${ }^{*}$ P. Ercius, ${ }^{* *}$ Abhay Raj Singh Gautam,** J. Cabana,* and U. Dahmen** \\ *Environmental Energy Technologies Division, Lawrence Berkeley National Laboratory, 1 \\ Cyclotron Road, Berkeley, CA 94720 \\ **National Center of Electron Microscopy, Lawrence Berkeley National Laboratory, 1 Cyclotron \\ Road, Berkeley, CA 94720
}

Despite the vast amount of research devoted to finding new materials to increase the energy density of Li-ion batteries, most of the devices available in the market still contain carbon as the negative electrode. Among the phases proposed to date as an eventual replacement, transition metal oxides $\left(\mathrm{M}_{\mathrm{x}} \mathrm{O}_{\mathrm{y}}\right.$, where $\left.\mathrm{M}=\mathrm{Mn}-\mathrm{Cu}\right)$ emerge as attractive candidates because they can store as much as twice the amount of charge per unit of mass than carbon electrodes [1]. Unlike the latter, which react with lithium through an intercalation mechanism, a conversion reaction leads to the formation of metallic nanoparticles embedded in a matrix of $\mathrm{Li}_{2} \mathrm{O}$, while the overall shape of the original crystal is retained after the reaction [2]. Nickel oxide (NiO) is an example of such a reactive conversion and is the system studied here. Because conversion is a slow reaction that entails large volume expansions and can induce particle strain and pulverization, nanoporous structures are best-suited for the goal of repeated reduction-oxidation cycling $[3,4]$. Nanoporous $\mathrm{NiO}$ can be synthesized by thermal decomposition of nickel hydroxide [5]. In this study, the porosity of nanoporous $\mathrm{NiO}$ to be used as battery electrodes was characterized using STEM tomography. Information obtained by tomography can be related to the kinetics and mechanism of the conversion reaction, and ultimately to the battery performance.

A tilt series with angles ranging from $-66^{\circ}$ to $66^{\circ}$ was acquired in STEM mode at $200 \mathrm{kV}$ and reconstructed using the sequential iterative reconstruction technique (SIRT). Before reconstructing, the images in the series were aligned using both cross-correlation and manual methods. The porosity and surface area of the crystal was measured using manual segmentation of the tomogram. The analysis of the 3D microstructure revealed several interesting details regarding the transformation of $\mathrm{Ni}(\mathrm{OH})_{2}$ plates to $\mathrm{NiO}$. The tomogram shows that the majority of the pores open on the plane of the plate while very few pores open on the faces around the perimeter of the plate. This results in a 'rim' of dense NiO surrounding the porous structure as shown in Figure 1a and also in the STEM and CTEM images of the $\mathrm{NiO}$ crystal (Figure 1b). These observations indicate that contrary to the mechanism suggested by the pseudomorphic and topotaxial nature of the transformation, the gas evolved during the decomposition process escapes mainly through, rather than parallel to the basal plane of the original hydroxide crystal.

The process of $\mathrm{Ni}(\mathrm{OH})_{2}$ decomposition during exposure to the electron beam was also observed directly by in-situ experiments. These experiments showed that a region of denser transformed material was formed along the perimeter of the crystal during the early stages of transformation, which forced the water molecules to eject along the $\mathrm{c}$ axis of the hydroxide crystal for the remainder of the process. The external volume of the crystal before and after decomposition was measured. The porosity calculated from the measured decrease in volume upon decomposition and the theoretical 
volume change obtained from the unit cell volumes of $\mathrm{Ni}(\mathrm{OH})_{2}$ and $\mathrm{NiO}$ were found to be in good agreement with the pore volume measured from the tomograms. These observations show that STEM tomography can provide reliable measurement of porosity in nanoparticles and provide useful insights in the understanding of phase transformations. In addition, electron diffraction and energy loss spectroscopy were used to obtain information on the mechanism of the transformation [6].

\section{References}

[1] J. Cabana et al., Adv Mater 22 (2010) E170.

[2] P. Poizot et al., Nature 407 (2000) 496.

[3] X. Wang et al., Cryst. Growth Des. 6 (2006) 2163.

[4] E. Hosono et al., Electrochem. Commun. 8 (2006) 284.

[5] L. Dong et al., Chem. Eur. J. 14 (2008) 5064.

[6] The authors acknowledge acknowledge support of the National Center of Electron Microscopy, Lawrence Berkeley Lab, which is supported by the U.S. Department of Energy under Contract \# DE-AC02-05CH11231.
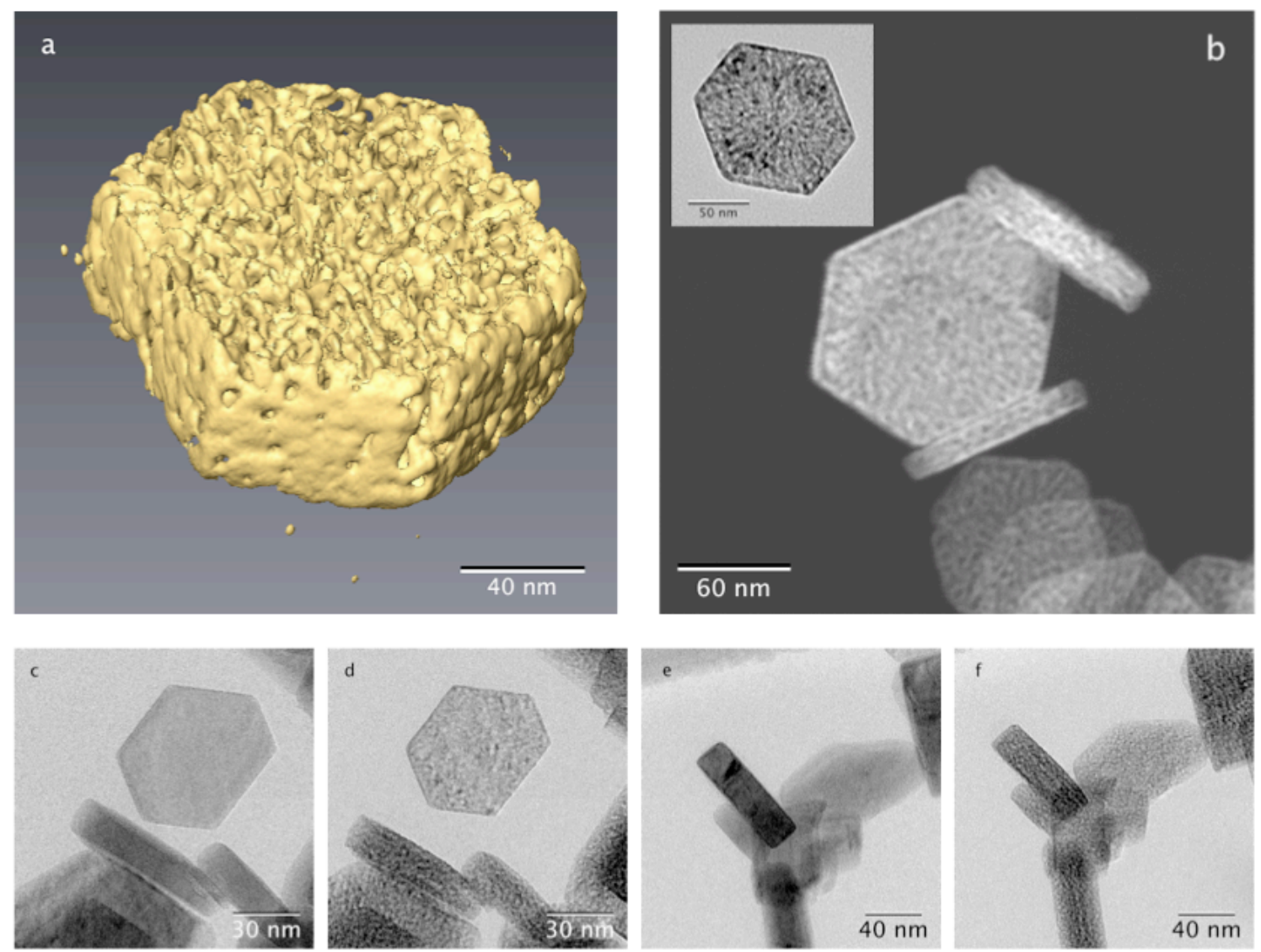

FIG. 1. (a) 3-dimensional view of the NiO crystal obtained by STEM tomography. (b) STEM and CTEM (inset) images showing a rim of denser material around the porous $\mathrm{NiO}$ crystal. (c) and (d) CTEM bright field image showing the face (close-packed plane) of $\mathrm{Ni}(\mathrm{OH})_{2}$ nanoplate and the transformed $\mathrm{NiO}$, respectively. (e) and (f) CTEM bright field image showing the thickness of $\mathrm{Ni}(\mathrm{OH})_{2}$ and the transformed $\mathrm{NiO}$, respectively. 